\title{
The experience with the design and refurbishment of the artificial sky in the SAS daylight laboratory
}

\begin{abstract}
Daylight laboratories are equipped with artificial skies for experimental research and testing daylight conditions in specific architectural models as well as for educational purposes. The latter often are meant only as attractive features to be shown to university visitors and students interested in architectural design or window applications but seldom are used for daylight research as measuring devices because these are not calibrated or are not modeling the standard ISO/CIE sky types.

During the sixtieth of the last century was designed and built in the SAS Daylight laboratory an artificial sky simulating the CIE Overcast and unity (Lambertian) skies and then the recently CIE Clear Sky with the artificial sun. The representation of sky luminance patterns as well as proportional parallel sunbeam modeling was designed and calibrated in documented private protocols. After forty years since the Bratislava artificial sky was in service this laboratory equipment became almost a historical remnant with its artificial lighting by incandescent reflectors and robust theatrical dimming system. New technology progress in artificial light sources, reduction of energy demands and computer checking possibilities as well as new knowledge in daylight science in the determination of new ISO/CIE sky types form new requirements for designing or refurbishing artificial skies. In this respect older experience and new ideas are summarised in this paper.
\end{abstract}

Keywords: Artificial sky, artificial sun, refurbishment and calibration requirements.

\section{Introduction}

Before designing the artificial sky with sun a review of artificial sky solutions [1] and a study about typical sky luminance distributions concerning $\mathrm{CIE}$ Overcast and $\mathrm{CIE}$ Clear Sky [2] were available. Considering the basic research and experimental purpose of the ICA SAS daylight laboratory as well as the design possibilities in the new Institute building at the SAS Patronka campus the new concept of the artificial sky with sun was realised [3], Figure 1. The main aims were following:

To simulate natural overcast, uniform and clear sky patterns with their smooth and fluent luminance changes a white reflective dome with a diameter 8 meters was chosen hanging on cables from the building roof construction;

- To model the artificial sun producing parallel beams a parabolic mirror with a $0.8 \mathrm{~m}$ diameter was installed on a trolley to enable its vertical movements adjusting a chosen solar altitude in the range from $0^{\circ}$ to $70^{\circ}$ as its maximum summer in Bratislava is $65.28^{\circ}$;

For the installation of the artificial lighting system illuminanting the dome were chosen two circular rows of incandescent reflectors were placed on the laboratory floor one directed to zenith for the gradual luminance rise on overcast skies, the second directed to horizon for clear sky simulations which was coupled with the third circumsolar ring of sources hidden behind the parabolic mirror that could model the scattering indicatrix influence;

- To represent any azimuth orientation of the architectural model placed on the measuring table it was on wheels on a circular rail track, thus any solar azimuth and model orientation could be adjusted by turning the table around;

In case of illuminance measurements in interiors were usually placed room models in a reduced scale 1:10 on the measuring table without a floor, thus photocells could be moved along grooves of the measuring table from underneath the table where researchers could take the measurements.

All these solutions proved to be very useful and effective due to calibration and practical experiments.

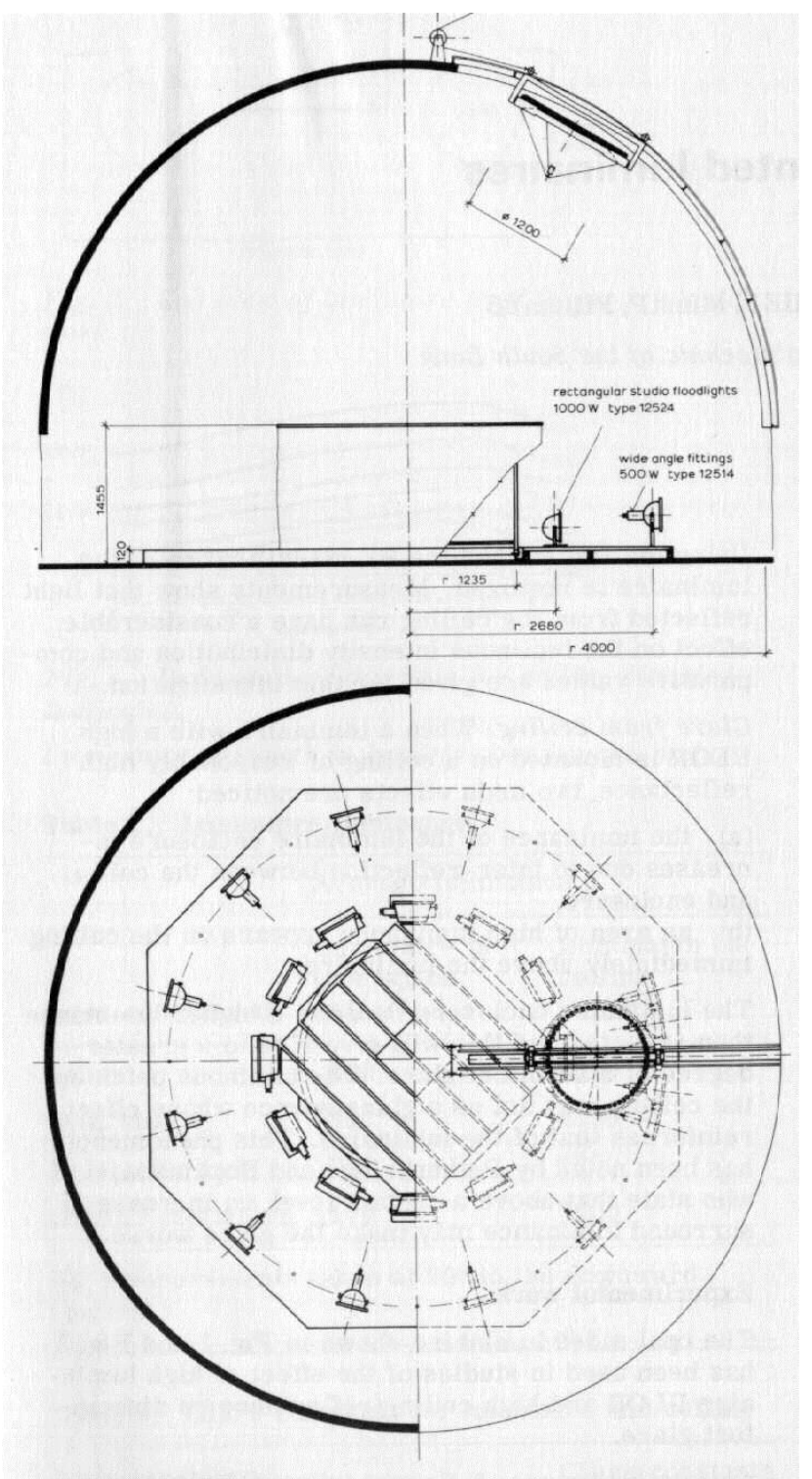

Fig. 1 Scheme of the artificial sky built in the ICA SAS in Bratislava 


\section{New requirements for present refurbishment}

Almost fifty years since the concept and design of the Bratislava artificial sky several new progress and demands have changed which have to be taken into account:

- Currently new laboratory instruments such as luminance and illuminance meters calibrated in SI units can respect smaller acceptance angles and scales;

- It is not possible to model extreme natural sun and sky luminance or illuminance levels in absolute physical units in an artificial sky, therefore their model scale ratios have to be calibrated and given in the calibration protocol;

- The advantage of artificial skies is in their time stableness so longer adjustments and calibration of typical sky luminance patterns such as ISO/CIE fifteen sky types [4] can be done properly;

- Current demands to reduce electrical energy by the artificial lighting system stimulate the change from the older incandescent to LED sources.

\section{The reasons for artificial sky refurbishment and its current state}

Recent progress and new knowledge in daylight science including the ISO/CIE standardisation of fifteen homogeneous sky types as well as the possibility of introducing sky luminance distributions and illuminance levels in physical units instead of relative ones, it is a substantial challenge when modelling artificial skies anew. If these facts are realised during the refurbishment design and solution the artificial sky can enable:

- Tests of architecturally complex interior models especially in case of the arbitrary obstructed windows;

- Experimental research of alternative applications of different shading devices, atrium and light well ducks or tubular guidelines under various ISO/CIE sky types;

- Laboratory measurements of innovative interior or façade designs by students or professional architects to demonstrate possible solutions in a trial to achieve better environmental and energy saving alternatives;

- Studies of window glare or insolation problems under clear skies in summertime or in tropics, etc.

For all these purposes the refurbished Bratislava artificial sky will serve to check theoretical hypothesis and computer calculation programs and in the calibration procedure will enable to equip the artificial sky with a set of energy efficient LED fittings.

The refurbishment work started after the project was accepted and granted financial support in October 2013 and several partial aims were already finalised, as:

- the dismounting of the artificial sun mirror and all reflectors of the old illumination system;

- a diffuse white paint was developed and produced by the Smolenice Chemolak s.a., tested by calibration and applied on the sky hemisphere interior surface;

a new special LED fitting was developed at the Dojc OMS Ltd. factory, tested in the reflective sky to enable further number of similar lamps to be produced;

the fish-eye camera was calibrated to measure luminance patterns;

- for calibration purposes a special tripod head adjustment was designed and made to enable simultaneous measurements by a luminance meter and the fish eye camera image; studies of ISO/CIE sky types with their simulations and intensity modelling proceeded and these results were already printed or offered for publication in several manuscripts (in References).

\section{The necessity of adequate sun and sky participation}

In many older artificial skies were reproduced only unity or the gradual CIE Overcast Sky with absolutely no direct sunlight. However, the absolute luminance and illuminance levels dependent on atmospheric transmission and various solar altitudes were also neglected. For the homogeneous atmospheric conditions under ISO/CIE standards the probably proportion between momentary sunlight and skylight was explained [5] and it is recommended to be respected in artificial skies. The mutual proportional participation of sunlight and skylight is dependent on the solar altitude, turbidity and cloud cover, type and spacing.

Due to the enormous space size of the sky vault representing the earth atmosphere a fictitious virtual hemisphere is imagined on which luminance ISO/CIE luminance patterns in small solid angles are simulated. It is important to model standard sky types in their homogeneous or quasi-homogeneous nature as in their mathematically defined set as proposed for daylight calculation purposes and basis for computer-aided design and evaluation tools. Therefore the simulation of the fifteen ISO/CIE luminance patterns in an artificial sky has to follow certain basic requirements and simulation rules such as:

A genuine evenness and fluent luminance patterns of all the fifteen ISO/CIE sky types have to be reproduced [6] in a certain model scale because real luminance in absolute units cannot be achieved in the artificial sky.

In the same scale have to be simulated also illuminance level on the measuring model table where architectural model buildings or room with windows will be placed.

- Due to the required precision of model measurements the hemispherical sky dome at least $7.5 \mathrm{~m}$ in diameter with a inner surface with high reflectance at least 0.9 coefficient is recommended to illuminate a model table representing an outdoor plane at least $2 / 2 \mathrm{~m}$ for placing the architectural model room with roughly 1/10-1/20 scale, Figure 2.

If the artificial sky is equipped also with an artificial sun producing parallel light beams then its size has to be a compromise between its distance from the model table, i.e. close to a small solid angle of the sun disc in reality and a contrary requirement to enclose all model windows with parallel sunbeams [7]. Furthermore, the interdependence of the sky dome luminance pattern with the intensity of sunbeams under different cloudy and clear sky types with typical atmospheric turbidity, i.e. luminous turbidity factor $T_{V}$ is to be simulated by dimming which needs a special attention in applying a suitable model scale with its calibration tests [8]. Levels of diffuse $E_{v, d}$, direct $E_{v, s}$ and global $E_{v, g}$ illuminances significantly depend on the and solar altitude, as is shown in Table 1.

- Besides the size of the artificial sun disc another problem is to be solved, i.e. to imitate the high solar corona luminance around it caused by the scattering indicatrix elongation towards the sun position, which is crucial under the clear sky luminance patterns. 


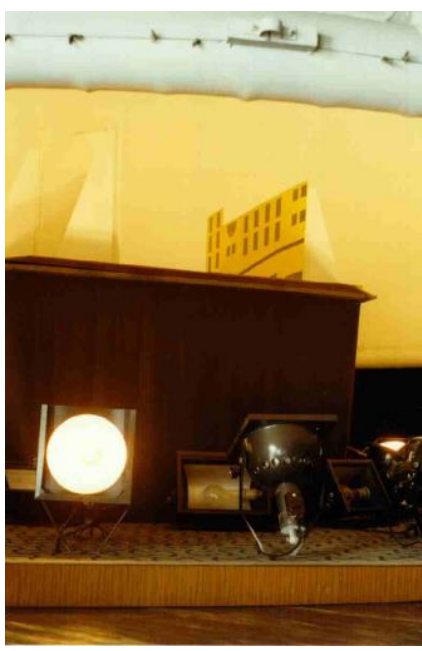

Fig. 2. Scale model on the table in the artificial sky

- When modelling the clear sky colour by LED sources it could be possible to consider also the simulation of usual bluish sky colour under clear sky conditions.

- In daylight research and model experiments main rules have to be respected in the scales of all models used.

Table 1. Simulation of exterior illuminance on selected clear skies

\begin{tabular}{|c|c|c|c|c|c|}
\hline Sky type & $T_{v}$ & Solar altitude & $E_{v, d}$ & $E_{v, s}$ & $E_{v, g}$ \\
\hline \multirow{4}{*}{12} & \multirow{2}{*}{2,5} & $30^{\circ}$ & 12,016 & 40,606 & 52,622 \\
\cline { 3 - 6 } & & $45^{\circ}$ & 15,117 & 66,369 & 81,486 \\
\cline { 2 - 6 } & \multirow{3}{*}{4,5} & $30^{\circ}$ & 19,626 & 27,235 & 46,860 \\
\cline { 3 - 6 } & & $45^{\circ}$ & 24,506 & 49,979 & 74,485 \\
\hline \multirow{4}{*}{13} & \multirow{2}{*}{3,5} & $30^{\circ}$ & 14,321 & 33,255 & 47,576 \\
\cline { 2 - 6 } & \multirow{2}{*}{5,5} & $45^{\circ}$ & 17,257 & 57,594 & 74,851 \\
\cline { 2 - 6 } & & $30^{\circ}$ & 24,060 & 22,304 & 46,365 \\
\hline & & $45^{\circ}$ & 28,287 & 43,371 & 71,658 \\
\hline
\end{tabular}

\section{Calibration methods applicable for artificial skies}

Although any simulated ISO/CIE sky type reproduced in the artificial sky in a certain scale is variable by different dimming of the chosen system of sources, once calibrated it can be fixed as stable for any long period and thus available for experimental work or tests in repeated cases.

Generally, there are possible three methods for calibrating artificial skies:

1. Utilizing a single portable luminance meter on an adjustable tripod with correct reading of vertical and horizontal angular directions can be used for measuring the relative gradation and scattering indicatrix functions [9] or partly as a sky scanner. Even the simplest artificial overcast skies have to be calibrated for vertical gradation as well as for testing azimuth evenness and symmetry in all directions, which can be distorted by the directional and space distribution of the output by electrical sources;

2. If a calibrated sky scanner is available this can be used in the procedure of repeated corrections of following stepwise changes of correcting directions, placement or dimming of electrical systems to reach the expected luminance patterns. However, due to the expensiveness of the sky scanner only few daylight laboratories own it in their regular photometric equipment;
3. Using a fish-eye camera with a $180^{\circ}$ acceptance angle is the probably best solution when this is calibrated to read any pixel luminance of the artificial sky. In fact a fisheye photo is representing an instant hemispherical sky image therefore it is more accurate than a scanner can catch due to its acceptance angle [10].

The presentation of a fisheye taken during clear day and its luminance analysis in the program LumiDisp [11] is compared with calculated standard luminance distribution in Fig. 3. Quite good coincidence of sky patterns is registered between these presentations.

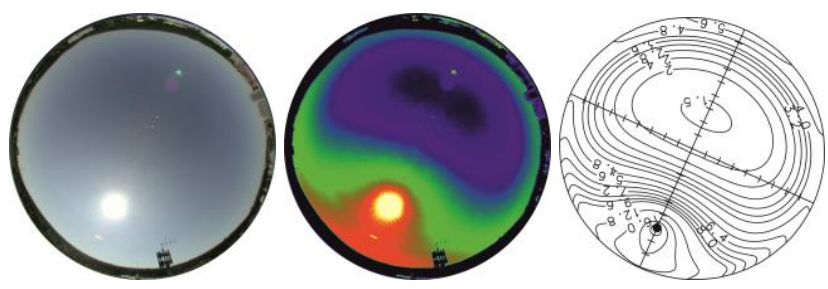

Fig. 3. Example of fisheye photo, luminance map and sky luminance interpretation by isolines

The calibration protocol for every artificial sky type has to state the descriptions of every used electrical circuit with the placement of all sources, their direction, dimming or other details.

\section{Conclusions}

After the preliminary study of older artificial skies with the artificial suns included the reconstruction of the forty years old Bratislava sky is justified. The concept and experience with the original design of structural elements has shown to be quite satisfactory excluding the expensive and robust dimming system of the reflector illumination scheme which needs updating. Its artificial illumination with incandescent sources has to be changed due to energy and inefficient colour rendering. At the same time a trial to simulate a new set of ISO/CIE standard sky patterns is possible not only in relative terms normalised to zenith luminance but also to adopt absolute physical units in the appropriate model scale reduction.

\section{Acknowledgements}

This study was done under the Slovak Research Grant APVV 0118-12 and the gratitude for this support is expressed.

\section{REFERENCES}

[1] Kittler, R.: An historical review of methods and instrumentation for experimental daylight research by means of models and artificial skies. Compte Rendu CIE Session, B, 319-334. Bureau Central, Paris 1960

[2] CIE - Commission Internationale de l'Éclairage : Standardisation of luminance distribution on clear skies. CIE Publ. 22. CB CIE Paris 1973

[3] Kittler, R.:A new artificial "Overcast and Clear Sky" with an artificial sun for daylight research. Lighting Res. Technol., 6 (1974), No. 4, 227-229

[4] ISO/CIE - International Standardisation Organisation or Commission International de l'Ěclairage: Spatial distribution of daylight - CIE Standard General Sky. ISO Standard 15409:2004

[5] Darula, S., Kittler, R. The simultaneous occurrence and relationship os sunlight and skylight under ISO/CIE standard sky types. Lighting Research Technology, DOI:10.1177/1477153514538883 
[6] Darula, S., Kittler, R. A methodology for designing and calibrating an artificial sky to simulate ISO/CIE sky types with an artificial sun. Leukos, 11 (2014), No. 2, 93-105

[7] Kittler, R, Darula, S. Relation between direct and diffuse daylight illuminance. Proc. Int. Conf. LUMEN V4, 8-10 Október 2014, Visegrád, 2014, 6 p.

[8] Darula, S, Kittler, R. Deriving illuminance for mode measurements under artificial skies. Proc. 28th CIE Session, Manchester, June 28 - July 4, 2015. In print.

[9] Kittler, R., Kocifaj, M., Darula, S. Daylight Science and Daylighting Technology, pp.115-118, Springer Science + Business Media, New York, 2012

[10] Souza, D.F., Scarazzato, P.S., Pedrini, H. Influencia da divisao do céu em calculos de disponibilidade de luz a partir de imagens de grande alcance dinamico. (In Portuguese: The influence of sky division in light availability calculations from High dynamic Range images.) XII Encontro Nacional e VIII Latinoamericano de Conforte no Ambiente Construido, paranoá 12, 2013
[11] Baxant, P., Sumec, S.; LumiDISP - software for the luminance distribution proccessing and digital photography analyses. Brno University of Technology, 2006; http://www.lumidisp.eu

Authors: Associate Professor Richard Kittler, DrSc., Institute of Construction and Architecture, Slovak Academy of Sciences, Dúbravská cesta 9, SK- 84503 Bratislava, Slovakia, e-mail: usarkit@savba.sk

Associate Professor Stanislav Darula, PhD., Institute of Construction and Architecture, Slovak Academy of Sciences, Dúbravská cesta 9, SK- 84503 Bratislava, Slovakia, e-mail:; email: usarsdar@savba.sk 